\title{
Principles of information organization in L2 use: Complex patterns of conceptual transfer
}

\author{
MONIQUE FLECKEN, CHRISTIANE VON STUTTERHEIM \\ AND MARY CARROLL
}

We will start the discussion of this special issue by placing the present domains of investigation within the broader context of the debate on languagespecificity in conceptualization processes. The debate has gained momentum in recent years, but central issues are still theoretically controversial and empirically inconsistent. The exact nature of the interrelation between language (in the sense of a specific language and specific linguistic structures) and the conceptual processes involved in language use is not clear. We can assume, on the basis of studies in this field and the focus on patterns of actual language usage, that the nature of this interrelation is highly dynamic in that it can differ across tasks, contexts, conditions and levels of complexity. The specific contribution of L2 studies to this debate is that it allows the comparison of processes of conceptualization which relate to the use of a specific language, to those of a different language, within an individual speaker. If cognitive processes during language tasks are somehow shaped by the categories of the respective mother tongue, speakers of a second language should - depending on the level of proficiency and the specific typological profile of the L1 (source language) and L2 (target language) - show divergent patterns from target standards with respect to the selection and organization of content for expression. Cook (1981) has already put the investigation of L2 users forward as a possible window on the language - cognition interrelation: "It would be highly useful if we could, so to speak, disengage the two processes of language and cognitive development and look at people whose level of thinking is out of step with their level of language (i.d second language learners)" (Cook 1981: 255; see also Bassetti and Cook 2011).

When focusing on the dynamics of the interrelation between language and cognitive processing, it is useful to draw on a language production model which spells out the different components and phases in the generation of a verbal representation of a given state of affairs. Levelt (1989) distinguishes three mod- 
ules, one being the conceptualizer in which a preverbal conceptual structure is generated, which is then passed on to the formulator in which the respective lexical, syntactic, and phonological forms are accessed, resulting in a linguistic structure which is then passed on to the articulator. In L2 production, transfer can occur at all of these levels. In the present context the focus of interest concerns transfer at the conceptual level. Levelt describes the tasks to be 'solved' by the conceptualizer as (a) macroplanning, which includes selection of information from a knowledge base, the linearization and structuring of information; (b) microplanning, which includes contextual embedding, selection of a referential frame, and selection of a specific perspective (Levelt 1989: Ch. 4). Although, in his original model, Levelt claims that these processes in the conceptualizer may not be language-specific, empirical results point in a different direction: Evidence points to the fact that, in the conceptualizer, the preverbal message has to be constructed on the basis of language-specific principles of information organization. These principles can be seen as a kind of filter on attention during a linguistic task, affecting the type of information selected and how this is structured at the macro- and micro-structural level (cf. von Stutterheim and Nüse 2003) (see graphic depiction of this entire process in a language production task, in Figure 1).

These principles in information organization can be traced, to a large extent, to the concepts and categories that have become lexicalized or grammaticalized in a specific language. Whereas lexical categories involving labels for objects (typically expressed by single words), for example, affect patterns in naming and, for example, performance on sorting or similarity judgement tasks, lexical or grammatical categories with scope over a number of different conceptual domains and larger linguistic units (an example of a lexical distinction: the semantic categorization of verbs, cf. Majid et al. 2008; examples of grammatical categories: tense, case marking), by contrast, play an important role in the establishment of principles in information organization. This holds in particular for grammatical categories that have gained obligatory status in specific linguistic systems (Bybee et al. 1994). Given the fact that grammaticalized means are obligatory in many contexts, accessing the forms and concepts associated with grammatical knowledge is highly automatized in native speakers. Cook (2011: 10) also points out that "there are, for instance, a host of cross-linguistic syntactic differences crying out to be tested against cognition" (see also Lucy 1992, for one of the first important studies looking at the effects of grammar on cognition). Preferences in structuring information may thus emerge given the grammatical encoding of specific conceptual distinctions in a given language (von Stutterheim and Nüse 2003; Schmiedtová et al. 2011). The complex nature of principles of information organization has implications for L2 acquisition and use. The notion of conceptual transfer, which so far has mainly been applied to lexicalized categories (cf. Pavlenko 2011: 246), has to be ex- 


\begin{tabular}{|c|}
\hline $\begin{array}{c}\text { Presentation of stimulus } \\
\text { Situation/event/story }\end{array}$ \\
\hline $\begin{array}{c}\text { Conceptualizer: } \\
\text { Message construction processes } \\
\text { Information organization at } \\
\text { macro-/micro-structural level }\end{array}$ \\
- processes of perspective taking \\
- segmentation, selection and structuring of \\
information \\
\hline $\begin{array}{c}\text { Formulator: } \\
\text { Formulation processes }\end{array}$ \\
grammatical and phonological encoding \\
- retrieval of word meanings and forms \\
construction of global and local syntactic \\
structure(s) \\
syllabification of sentence structure
\end{tabular}

Figure 1. Language production process involving three different modules (based on Levelt 1989; Griffin and Bock 2000), in the context of event or narrative construal, given a visual stimulus (task instructions "tell what is happening in the stimulus").

tended to this highly abstract level of conceptualizing information for expression.

For a language user, solving a complex task, such as in sentence or text production, means that the following sub-tasks have to be solved in detail during the process of conceptualization (see also Figure 1): a) a relation to context has to be established (reference frames), b) relevant conceptual units have to be selected, c) semantic relations across these units have to be established, as well as between units of the same domain across sentences, and d) information status has to be assigned to these units (topic-focus) within and across sentences. Any language user has to solve these different tasks, with due speed in language production and comprehension. These different tasks will have to be fulfilled in an integrated fashion, when planning how an event, for example, will be linguistically represented in a production task, or when interpreting certain functional relations in comprehension. The interplay of several different factors is highly complex and opaque, and success in acquisition means that 
the L2 learner has to disentangle this when processing the input. For example, it may be one thing to acquire the forms of a grammatical category such as progressive aspect (to be -ing in English, is aan het V-inf in Dutch), however, the identification of principles according to which progressive aspect is actually used, and an understanding of the function of this morphological marker in a stretch of discourse in a specific language is a different matter. It is this level of linguistic knowledge with which the papers in the present volume are concerned.

One problematic issue lies in the fact that we do not fully understand how all of this works in a person's native language, as it has not been given much attention in linguistic or psycholinguistic research in recent years. All of the present papers also provide indepth analyses of the performance of native speakers on a variety of tasks, in order to set an appropriate baseline for the L2 users. Highly advanced L2 speakers who have adult cognitive capacities and the linguistic repertoire for fulfilling complex communicative tasks are very likely to draw on L1-based principles of information organization. The central research questions can be listed as follows; a) empirical: how does the learner proceed (L1-oriented, L2-oriented or on the basis of learner-specific structures or strategies?), and b) explanatory: what components of the L2-based principles does a learner acquire, at what stage of proficiency and degree of L2 exposure and use, and by which intermediary steps? Studies that address the level of complexity associated with L2 learning of such knowledge are scarce. It is the purpose of this special issue to contribute to these questions, focusing specifically on the conceptual implications of grammatical structures, during production or comprehension tasks. The present collection of studies encompasses a range of tasks and methods and focuses on a number of different languages and language combinations (both native speakers and L2 users), shedding light on issues of language typology (e.g., the role of typological distance in L1-L2 pairs on cognitive processing, see below). We will first list the domains and levels of investigation in the present collection of empirical papers. This is followed by a discussion of the findings on the different samples of L2 users in terms of conceptual restructuring ('restructuring' is used as a term covering several options in changes in speakers' conceptual categories and processing patterns, cf. Pavlenko 2005, 2011; Jarvis and Pavlenko 2008). Finally, the present studies will be related to previous L2 research in the same context, and we conclude with a list of the main factors that contribute to the patterns obtained.

\section{Tasks, domains and levels of $L 2$ performance}

Two papers in the present special issue address the role of the linguistic category of grammatical aspect (progressive, imperfective, perfective) in event 
construal, with analyses based on descriptions of sets of single events of different types, as depicted in dynamic stimuli. Schmiedtová focuses specifically on motion events for two languages (Czech and English), which show the following differences in perspective taking: motion events are preferably presented holistically with mention of an endpoint in Czech, and events with a resultant state are marked as completed by means of perfective aspect; this contrasts with English where events are segmented into phases, given the use of progressive aspect, with a focus on the phase which is currently in progression. The analysis of advanced Czech learners of English, on the basis of the same stimuli, illustrates how the Czech learners of English display transfer at the level of perspective taking, involving the perfectivity of an event, an aspectual distinction that cannot be marked morphologically in English. It is is expressed in the L2 by a more elaborate focus on the final phase of an event, encoded lexically. Although the learners are highly proficient, the patterns for information selection still reflect those of the native language. Schmiedtová also discusses the results of an eye tracking study which provides supporting evidence for this form of perspective taking. The results thus present instances of conceptual transfer.

The analysis by van Beek et al. covers single events from three situation types (dynamic stimuli showing causative actions, activities, motion events) and concerns the role of progressive aspect in marking the specificity of an event, comparing L1 Dutch and L1 German speakers with very advanced German learners of Dutch. The specificity of an event can be encoded by means of progressive aspect (anchoring the event in the here and now), as in L1 Dutch (een man is aan het breien 'a man is knitting'), or in its absence, as in L1 German, by referring via lexical means to specific features of the agent or the action in a causative event (i.e., events with an agent performing an action on an object; ein junger Mann strickt einen bunten Schal 'a young man knits a colourful scarf'). Although their event descriptions do not display any lexical or grammatical errors, the L2 Dutch speakers do not use progressive aspect to the same extent as Dutch native speakers. In contrast to L1 Dutch speakers, they are overexplicit in mentioning features of the agent depicted, reflecting L1 German, but they are also specific in relation to the action. While an elaborate focus on the agent in marking specificity can be interpreted as showing potential influence of the L1 on the L2, the focus on the action may also be interpreted as partial restructuring of perspective taking, even though the means used in L2 Dutch are lexical (phrases encoding specific elements of the action), whereas they are grammatical in L1 Dutch (a high frequency of use of progressive aspect). Findings are discussed in terms of the difficulty in acquisition when the input for learners does not display clear-cut patterns: Progressive aspect, though frequently used in the situation types in question, is still optional in Dutch, given its stage of grammaticalization. Although the findings 


\section{Monique Flecken, Christiane von Stutterheim and Mary Carroll}

in van Beek et al. illustrate partial adherence to the target language system, the overlap between Dutch and German with respect to aspectual perspective taking, i.e., the fact that it is perfectly acceptable in Dutch to describe events without progressive aspect, may have made it easier for German learners to adhere to the pattern that resembles their native language most (their event descriptions in Dutch thus display a lower frequency of aspect marking). However, the fact that the learners do not mainly rely on aspect to mark specificity may also reflect that for the learners the progressive form concerns aspect, first and foremost, and with this a specific perspective on the event - its being in progression. Its implications at the level of tense (i.e., explicitly relating the assertion to the here and now and thus encoding the event as a specific case) may possibly come into focus at a later stage in language acquisition. This would provide an example of an internal logic, so to speak, in specific domains, and how this drives the course of acquisition.

Two papers relate to information organization in narratives (stories), elicited from both L1 and L2 speakers, and are based on silent animation films. In the study of L1 German, L1 Japanese and L2 German learners of Japanese, Tomita uses a highly controlled experimental design in which specific scenes allow the elicitation of means required when linking events on a contrastive or additive basis in narratives. The cross-linguistic comparison is based on contrasts in the domains time and entities which are relevant for information organization in narratives. Tomita investigates the implications of scope particles (contrastive and additive) for information organization at the macro-structural level. In her comparison of native speakers, she shows that Japanese speakers prefer a coherence pattern which is based on relations between elements of the type 'entities' (preferably in subject position), whereas German speakers rely on temporal relations in establishing coherence in narrative texts. These differences at the level of information organization are rooted in lexical and syntactic properties of functional particles and syntactic constraints on word order in the respective language, and they imply language-specific constraints on information structure. This means that an L2 learner has to detect principles which are not apparent with respect to a clear-cut form-function relationship in the target language, but which operate on the level of the conceptualization of information for speaking. The learner data show that the L2 speakers use the new forms on the basis of a hybrid pattern, combining components of the L1 (temporal perspective) and L2 (entity-based coherence). This pattern may be interpreted as showing 'convergence' in the sense of Jarvis and Pavlenko (2008). On the basis of a short silent film, the study by Natale relates to spoken narratives by samples of Italian and French native speakers, as well as early and late French-Italian bilinguals (high-intermediate). The focus is placed on linkage (subordination, coordination) in narratives by early and late French Italian bilinguals with regard to preferences in topic management (Italian is a 
null-subject language, whereas French is not). Italian displays patterns in subordination that serve to support topic maintenance with a null-subject, while French does not. Although the early bilinguals use the relevant means that support topic maintenance (gerunds) to a higher extent than the late bilinguals, both groups use subordinate clauses that 'disrupt' topic maintenance, reflecting the French pattern. In the analysis of coordination, Natale identifies the learners' reliance on core principles in information organization in narratives, as the learners show use of temporality and linkage in the form of temporal relations, which is not observed in L1 Italian or L1 French. Findings for the late bilinguals thus show partial L1 transfer of linkage patterns, and in the case of early bilinguals, an effect that resembles 'convergence' of linkage patterns, as well as a reliance on general principles, i.e., temporality as a core domain in structuring individual events within a narrative sequence.

Ellert provides findings from an eye tracking study on the comprehension of ambiguous pronouns by Dutch learners of German at two proficiency levels. The study uses the visual world paradigm and looks at points in time at which the ambiguity seems to be 'resolved'. A comparison is drawn between native speakers of German and two groups of Dutch learners of German. The study presents evidence of a general principle of information organization in the L2 users, an overall topic preference, also identified in the 'basic variety' for early as well as later stages of L2 acquisition (cf. Klein and Perdue 1997). This pattern emerges despite typological similarity in this regard between Dutch and German, and thus manifests a unique learner-specific pattern.

\section{Conceptual 'restructuring' in L2 users/bilinguals}

Two main types of generalisations can be drawn on the basis of the results obtained in the different studies in this issue:

(1) When comparing message generation between native speakers of different languages on the basis of the same verbal task, we find different patterns in information selection, information structure and in the choice of referential frames and the selection of perspective. Evidence shows that these patterns are constructed on the basis of principles which operate globally, at sentence and text level, and involve processing at the level of conceptualization (see Figure 1 above). They provide the basis for the integration of constraints across different conceptual domains and across the different levels of the message generated, or interpreted (semantic, syntactic, pragmatic).

(2) Advanced L2 speakers, when conceptualizing content for speaking, or when interpreting content in linguistic form, do not follow either L1 or L2 principles. They identify and apply only certain components and im- 
plications of the L2 principles, and combine them with components of L1 principles that may have a comparative range of application. Some findings point to the fact that L2 users rely on learner-specific strategies, which involve a simplification of the task, in that they adhere to basic principles in information organization (e.g., time or temporality in narrative structure), without realizing the intricate interplay and hierachical weighting between and within different conceptual domains.

A comprehensive framework of patterns in conceptualization in L2 users and bilinguals (in verbal and non-verbal tasks) is provided in Pavlenko $(2005,2011)$ and Jarvis and Pavlenko (2008). L2 performance can signal (1) the coexistence of L1 and L2 patterns of conceptualization, (2) the transfer of L1-based patterns, (3) a convergence of L1 and L2 patterns, leading to a 'unique' or 'in between' pattern of performance, (4) the restructuring of conceptualization patterns, (5) the internalization of new patterns of conceptualization, (6) the influence of the L2 on L1 performance, and (7) the attrition of L1 patterns. Studies in the present special issue focus only on performance in the second language of the bilingual speakers, and thus cannot make claims with respect to (1), (6) and (7). In the current collection of studies, we find evidence for the following patterns: (a) (Partial) transfer of viewpoint or perspective-taking patterns from the L1, at sentence level (Schmiedtová, van Beek et al., this issue); (b) (Partial) transfer of macro-structural principles of information organization (Natale, Tomita, this issue), leading to a type of 'convergence' between L1 and L2 patterns; (c) General learner-specific patterns in information organization (Ellert, Natale, Tomita, this issue). All in all, findings for all L2 samples display complex patterns of combinations of L1 and L2-specific principles. The patterns and principles we investigate represent an interplay of concepts, linguistic form and processing and attentional processes. Such patterns are not easy to restructure, and it is an empirical question, whether they ever can be restructured fully in L2 acquisition.

The central research question following from this classification of potential patterns of conceptualization, and the underlying principles in bilingual minds, concerns the factors which determine the dynamics of maintenance, expansion and reduction of the relevant L1 principles and the interactions with newly established L2 patterns. In other words, what are the driving forces behind (1) to (7) and the patterns found in the present collection of studies, and in what contexts do these patterns occur in the $\mathrm{L} 2$ performance of an individual? One set of factors which plays a role in this context concerns the specific linguistic and sociocultural variables that may affect the performance of a specific language user, on a specific task, at a specific time (e.g., degree and length of exposure, age of acquisition, length of residence in L1 or L2 speaking country, etc., see Athanasopoulos 2011). A further group of factors relevant in under- 
standing the dynamics of L2 performance is given by the requirements of the specific conceptualization process: the level of complexity and the specific task demands of the linguistic phenomena investigated, anything between the word level and the level of complex macro-structural networks, and the level of contextual embedding involved, given a specific linguistic task. We also need to take into account the degree of automaticity in drawing on a particular principle of information organization; principles that are rooted in the grammar of a language may be particularly difficult to restructure, in that they are activated automatically in a given context. The papers in the present volume indeed show how the type of knowledge that is associated with principles in information organization is highly automatized and deeply rooted in native language patterns of speech and processing.

\section{Conclusions: The bilingual mind}

In conclusion, we would like to focus attention on a set of explanatory factors (besides speaker-specific characteristics, see Athanasopoulos 2011), that are relevant for L2 performance and processing, taking into account studies in this issue, as well as previous work on the interplay between the two languages at the level of the conceptualizer within a bilingual mind:

(a) The level of complexity of a given linguistic task and associated task demands (for example, the contextual factors and conceptual domains involved).

In previous studies, we find strong conclusions, stating, for example, that the two languages of a bilingual are nearly always activated, and that lexical access in production and comprehension is nearly always non-selective. Existing models of bilingualism, for example, the Revised Hierarchical Model (e.g., Kroll and Stewart 1994; Kroll and Tokowicz 2005; see recent discussion of the RHM in Brysbaert and Duyck 2010), address issues such as conceptual organization and conceptual access, on the basis of research on single word production and comprehension mainly, thus predominantly focusing on the lexicon. In this domain evidence has been found for direct conceptual access in the L2, given a high level of proficiency. However, fine-grained differences in the types of conceptual distinctions that are made in one language, but not, or slightly differently, in the other language of a bilingual speaker, are not addressed. In other words, findings are interpreted as showing a high degree of flexibility of the cognitive and linguistic system, without addressing word-to-referent mapping in L2 users in specific cases in which concepts are not translatable or difficult to translate (see also Pavlenko 2011). Furthermore, linguistic principles relating to the organization of information at sentence or text level have not been at the main focus of attention. 
Studies that do explicitly investigate language-specific concepts, at the level of single words, show patterns of restructuring in naming, as well as in performance on non-linguistic tasks (see, for example, Athanasopoulos (2009) on the categorization of colour by bilinguals at different proficiency levels, showing clear effects of this variable), or evidence for a pattern of (partial) 'convergence' in naming objects (for example, Ameel et al. (2005) on simultaneous bilinguals). Studies on event conceptualization and narrative structure, in addition to the present studies - see, for example, Flecken (2011a), Schmiedtová et al. (2011) and Carroll et al. (2012), all addressing the conceptualization and description of events, as well as Carroll and Lambert (2003; 2006), Flecken (2011b) and Benazzo et al. (2012), looking at the construal of narratives find either evidence of the persistence of L1 patterns in performance in the L2 (transfer), or partial adherence to target-like patterns, or a pattern which relies on core principles of information organization (as in some of the studies in the present volume, as well). All studies look at highly proficient L2 users.

We will illustrate the complexity of the patterns identified in L2 performance with an example, given by a study on the conceptualization of motion events in verb-framed and satellite-framed languages (e.g., French versus English and German), and the acquisition of these contrasts in an L2 (Carroll et al. 2012). The study shows that the crucial difference for the French L2 learners of German and English is the fact that directional concepts are encoded in the verb in French (the learners' L1) and they are derived from the entity in motion (its orientation and distance towards a potential goal, e.g., se diriger vers; an entity is heading towards (a goal), directing him-herself to (a goal)), while in English and German directional concepts are drawn from the ground and its contours (e.g., an entity moves along the road, around the bend), as encoded in satellites/adjuncts (e.g., prepositional phrases). The information in the verb in English and German is confined to the manner in which the entity moves, but not its direction, and although L2 English speakers correctly use manner verbs, the adjuncts with which they combine them in sentences specify the location of the moving entity (e.g., a person is walking on a road). This focus on the location of the entity is not the pattern in the target languages, in which manner verbs are conjoined with adjuncts that specificy the path taken by the entity via contours of the ground (walking along a road/around a bend), on its way to a goal. The L2 speakers thus transfer the core component of the L1 French principle, in which the moving entity with its specific spatial features (e.g., oriented towards a potential goal, or located in a specific place) provides the anchor in event conceptualization for motion events. Significantly, this shows that attention in L2 production of these advanced learners is still directed to features of the moving entity as the source for directional information, and not to the ground as in satellite-framed languages (also confirmed in an eye tracking study currently in progress; see also attentional patterns in eye track- 
ing in similar L2 studies von Stutterheim and Carroll 2006; Flecken 2011a; Schmiedtová et al. 2011). Given that means that relate to the contours of the ground also exist in the learners' L1 (French), the findings thus pinpoint the role of attentional processes when processing information for expression, and the complexity involved in restructuring knowledge of this type for the learner.

In some instances, L2 influences on L1 performance have been reported in domains with a similar high degree of complexity, as in event and narrative construal (Bylund 2009; Brown and Gullberg 2010; Bylund and Jarvis 2011, etc.), given extensive exposure to the L2. These findings again reveal that the level of complexity and the interrelation of specific conceptual domains is something that will have to be addressed, when drafting models on conceptual access and organization in bilinguals.

So where do we stand? We stand at a point in time at which we should go beyond focusing on basic concepts and conceptualizations at the word level in L2 acquisition studies, and address more realistic patterns of language usage and processing in real time, and see how this type of knowledge, associated with either L1 or L2, is accessed and used during the course of L2 acquisition. Studies on language production present evidence for the fact that this type of knowledge is stored at a conceptual level, and how it may influence attention and processing during very early phases of production, thus showing the depth of entrenchment of this knowledge (see a large cross-linguistic study using eye tracking, von Stutterheim et al. 2012). We thus find that specific linguistic structures, in particular grammatical structures, focus speakers' attention on specific conceptual domains, and the features associated with them - they determine perspective taking and the viewpoint selected for structuring information at sentence or text level. The acquisition of this type of knowledge clearly goes beyond the acquisition of form alone. Evidence shows that L2 performance is definitely not erroneous, but relies on different underlying principles. In some cases direct transfer from L1 patterns can be identified. In other cases, the picture obtained seems much more complex, with evidence for partial L1 transfer of conceptualization patterns, a focus on basic principles of information organization and merged, mixed or convergent systems.

Further important factors relate to:

(b) The specific typological profile of both the L1 and L2, and the extent to which lexicalized and grammaticalized categories, with their scope for decisions made at the macro- and micro-structural level, may overlap or differ; the extent to which principles of information organization may overlap or differ.

With regard to the role of the typological distance between the two languages of a bilingual speaker, the findings in the present collection of papers present a diverse picture. Two of the papers cover learners acquiring a language which 
is typologically far removed from their native language, as for example in the case of German speakers learning Japanese, and Czech speakers learning English as an L2. Three other papers look at the performance of L2 users in a language which is typologically close to their native language, as in the case of Dutch speakers acquiring German, German speakers acquiring Dutch and French speakers acquiring Italian. At a first glance, there are no major differences in the outcomes of L2 acquisition as investigated in the individual papers. In nearly all cases, there is evidence for (at least, partial) transfer of native language principles. The studies on learners of typologically close languages specifically also provide evidence for learner-specific strategies or principles. The Dutch learners of German in Ellert's paper show patterns of pronoun resolution that do not resemble L1 or L2 patterns - even given similar patterns in Dutch and German native speakers. In van Beek et al. we find that the German learners of Dutch transfer L1 patterns of perspective taking in part only, i.e., an elaborated focus on agent features, but they also use progressive aspect to some extent, thus reflecting the Dutch pattern. The overlap between Dutch and German with respect to aspectual perspective taking, i.e., the fact that it is perfectly acceptable in Dutch to describe events without progressive aspect, may have made it easier for German learners to adhere to the pattern that resembles their native language most. The Czech learners of English (Schmiedtová, this issue) display transfer in perspective taking, not reflected in the use of aspectual morphology, as perfective aspect is not a morphological marker in English, but through an elaborate focus on specific phases of an event (final phases). This shows that although the learners are highly proficient, their patterns in information selection still reflect native language principles. All studies provide elaborate analyses of L1 use, thus going beyond predictions of L2 performance made on the basis of simple typological classifications.

In general, we find that conceptual transfer and conceptual restructuring is dynamic and dependent on the specific context and demands associated with the linguistic task - besides speaker-specific characteristics. Complex principles in information organization based on the L1 can be partially 'restructured' resulting in a hybrid structure. Such principles are related to "conceptual networks", so to speak, and require the integration of knowledge from several different domains and form-function relationships. Information processing of this type is highly automatized in native speakers and this type of processing seems to take place at a conceptual level. There is a high potential in L2 acquisition for the transfer of attention-directing concepts in information organization; as this type of transfer involves conceptual networks, something we may label "conceptual network transfer", given the network status of all the levels involved. Patterns of "conceptual-network transfer or restructuring" are affected by an interplay of the factors discussed above. The acquisition of this type of complex knowledge, and the automaticity of processing patterns asso- 
ciated with accessing this knowledge, represents the ultimate challenge for any L2 learner.

\author{
Radboud University Nijmegen \\ $\langle$ m.flecken@donders.ru.nl $\rangle$ \\ Heidelberg University \\ 〈stutterheim@idf.uni-heidelberg.de〉 \\ 〈carroll@idf.uni-heidelberg.de〉
}

\title{
References
}

Ameel, Eef, Gert Storms, Barbara C. Malt \& Steven A. Sloman. 2005. How bilinguals solve the naming problem. Journal of memory and language 53. 60-80.

Athanasopoulos, Panos. 2009. Cognitive representation of colour in bilinguals: The case of Greek blues. Bilingualism: Language and cognition 12(1). 83-95.

Bassetti, Benedetta \& Vivian J. Cook. 2011. Relating language and cognition: The second language user. In Vivian Cook \& Benedetta Bassetti (eds.), Language and bilingual cognition, 143-190. New York: Psychology Press.

Benazzo, Sandra, Cecilia Andorno, Grazia Interlandi \& Cédric Patin. 2012. Perspective discursive et influence translinguistique: Exprimer le contraste d'entité en français et en italien L2. LIA 3(2). 173-201.

Brown, Amanda \& Marianne Gullberg. 2010. Changes in encoding of path of motion after acquisition of a second language. Cognitive linguistics 21 (2). 263-286.

Brysbaert, Marc \& Wouter Duyck. 2010. Is it time to leave behind the revised hierarchical model of bilingual language processing after fifteen years of service? Bilingualism: Language and cognition 13(3). 359-371.

Bybee, Joan L., Revere Perkins \& William Pagliuca. 1994. The evolution of grammar. Chicago: Chicago University Press.

Bylund, Emanuel. 2009. Effects of age of L2 acquisition on L1 event conceptualization patterns. Bilingualism: Language and cognition 12 (3). 305-322.

Bylund, Emanuel \& Scott Jarvis. 2011. L2 effects on L1 event conceptualization. Bilingualism: Language and cognition 14(1). 47-59.

Carroll, Mary \& Monique Lambert. 2003. Information structure in narratives and the role of grammaticised knowledge: A study of adult French and German learners of English. In Christine Dimroth \& Marianne Starren (eds.), Information structure and the dynamics of language acquisition, 267-287. Amsterdam: Benjamins.

Carroll, Mary \& Monique Lambert. 2006. Reorganizing principles of information structure in advanced L2s: A study of French and German learners of English. In Heidi Byrnes, Heather D. Weger-Guntharp \& Katherine Sprang (eds.), Educating for advanced foreign language capacities, 54-73. Georgetown: Georgetown University Press.

Carroll, Mary, Katja Weimar, Monique Flecken, Monique Lambert, Christiane von Stutterheim. 2012. Tracing trajectories: motion event construal by advanced L2 French-English and L2 French-German speakers. LIA 3(2). 202-230.

Cook, Vivian J. 1981. Some uses for second language learning research. Annals of the New York Academy of Sciences 379. 251-258.

Cook, Vivian J. 2011. Relating language and cognition: The speaker of one language. In Vivian J. Cook \& Benedetta Bassetti (eds.), Language and Bilingual Cognition, 3-22. New York: Psychology Press.

Flecken, Monique. 2011a. Event conceptualization by early bilinguals: Insights from linguistic and eye tracking data. Bilingualism: Language and cognition 14 (1). 61-77. 


\section{Monique Flecken, Christiane von Stutterheim and Mary Carroll}

Flecken, Monique. 2011b. Macro-planning in narratives: Assessing bilingual attainment. International journal of bilingualism 15(2). 164-186.

Griffin, Zenzi \& Kathryn Bock. 2000. What the eyes say about speaking. Psychological Science 11. 274-279.

Jarvis, Scott \& Aneta Pavlenko. 2008. Crosslinguistic influences in language and cognition. London: Routledge.

Klein, Wolfgang \& Clive Perdue. 1997. The basic variety (or: Couldn't natural languages be much simpler?). Second language research 13. 301-347.

Kroll, Judith F. \& Erika Stewart. 1994. Category interference in translation and picture naming: Evidence for asymmetric connections between bilingual memory representations. Journal of memory and language 33. 149-174.

Kroll, Judith F., \& Natasha Tokowicz. 2005. Models of bilingual representation and processing. In Judith F. Kroll \& Annette M. B. de Groot (eds.), Handbook of bilingualism: psycholinguistic approaches, 531-553. Oxford: Oxford University Press.

Levelt, Willem. 1989. Speaking: From intention to articulation. Cambridge: MIT Press.

Lucy, John A. 1992. Grammatical categories and cognition: A case study of the linguistic relativity hypothesis. Cambridge: Cambridge University Press.

Majid, Asifa, James Boster \& Melissa Bowerman. 2008. The crosslinguistic categorization of everyday events: A study of cutting and breaking. Cognition 109. 235-250.

Pavlenko, Aneta. 2005. Emotions and multilingualism. Cambridge: Cambridge University Press.

Pavlenko, Aneta. 2011. Thinking and speaking in two languages. Clevedon: Multilingual Matters.

Schmiedtová, Barbara, Christiane von Stutterheim \& Mary Carroll. 2011. Language-specific patterns in event construal of advanced second language speakers. In Aneta Pavlenko (ed.), Thinking and speaking in two languages, 66-107. Clevedon: Multilingual Matters.

Stutterheim, Christiane von \& Ralf Nüse. 2003. Processes of conceptualization in language production: Language-specific perspectives and event construal. Linguistics 41(5). 831-881.

Stutterheim, Christiane von \& Mary Carroll. 2006. The impact of grammatical temporal categories on ultimate attainment in L2 learning. In Heidi Byrnes, Heather Weger-Guntharp, \& Katherine A. Sprang (eds.), Educating for advanced foreign language capacities, 40-53. Georgetown: Georgetown University Press.

Stutterheim, Christiane von, Martin Andermann, Mary Carroll, Monique Flecken \& Barbara Schmiedtová. 2012. How grammaticized concepts shape event conceptualization in language production: Insights from linguistic analysis, eye tracking data and memory performance. Linguistics 50(2). 833-867. 